\title{
Assessing Graduate Admissions Metrics
}

\author{
A statistical analysis suggests that among the metrics used for admissions \\ to US graduate programs in physics, grade point average exhibits the \\ strongest correlation with indicators of academic success.
}

\author{
By Matteo Rini
}

W hen university departments pick students for their doctoral programs, they often do it with the aid of quantitative metrics. Recent research, however, suggests that some widely used metrics are unreliable predictors of academic success and disproportionately disfavor women and underrepresented minorities. Mike Verostek of the University of Rochester, New York, and colleagues have now used statistical analysis methods to compare two metrics used in the US and Canada-undergraduate grade point average (GPA) and the Graduate Record Examinations (GRE) [1]. They find that only GPA correlates well with a set of indicators of graduate performance.

Undergraduate GPA is determined by nonstandardized tests put together by individual schools. The GRE was introduced 85 years ago to offer a more standardized indicator for assessing certain analytical skills and, for the physics GRE, specific knowledge in the field. Recently, however, schools have been

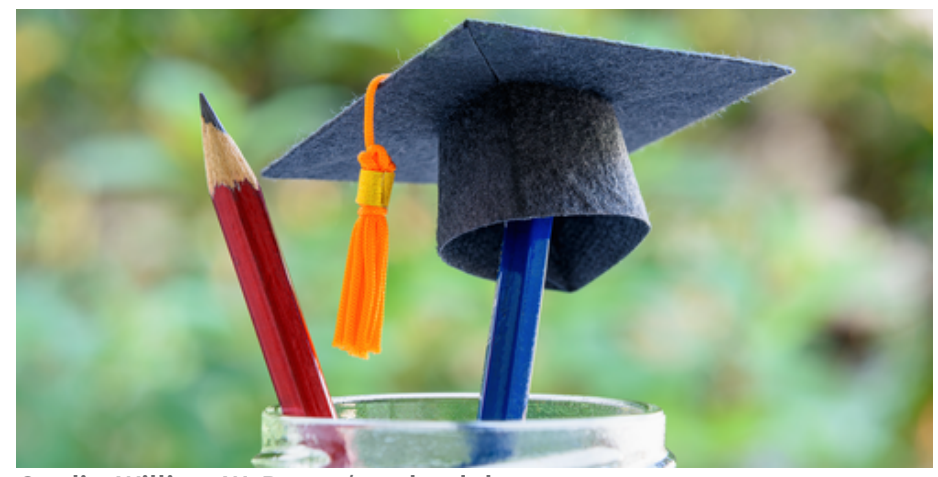

Credit: William W. Potter/stock.adobe.com increasingly dropping their use of the GRE. These decisions are based on studies, such as this recent one by some of Verostek's colleagues, suggesting that the tests are unreliable predictors of a person's future success in graduate school.

In their analysis, Verostek and his colleagues compare how well undergraduate GPA and GRE scores correlate with a broader set of "success" indicators than those considered in the previous studies. They find that compared with GRE scores, undergraduate GPA more strongly correlates with both Ph.D. completion and graduate GPA. The researchers say that their finding strengthens the case that undergraduate GPA is a more useful and equitable metric than the GRE, but they also advocate for a more holistic admission process that accounts for factors that no metric can possibly quantify (see Opinion: Equitable Admissions in the Time of COVID-19).

Matteo Rini is the Editor of Physics.

\section{REFERENCES}

1. M. Verostek et al., "Analyzing admissions metrics as predictors of graduate GPA and whether graduate GPA mediates Ph.D. completion," Phys. Rev. Phys. Educ. Res. 17, 020115 (2021). 